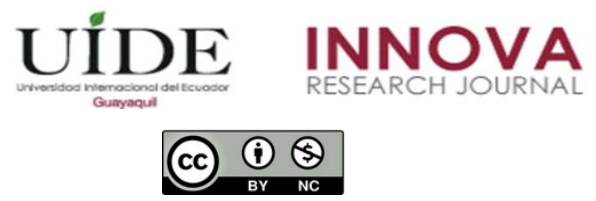

INNOVA Research Journal, ISSN 2477-9024

(Septiembre-Diciembre 2020). Vol. 5, No.3.1 pp. 329-346

DOI: https://doi.org/10.33890/innova.v5.n3.1.2020.1542

URL: http://revistas.uide.edu.ec/index.php/innova/index

Correo: innova@uide.edu.ec

\title{
Desbalance entre costo de inventario y nivel de servicio de producto terminado
}

\section{Unbalance between the cost of inventory and the level of service of the finished product}

Diego Paúl Quezada Cepeda

Investigador Independiente, Ecuador

Santiago Nicolás Nájera Acuña

(D) https://orcid.org/0000-0001-5972-1556

Universidad Internacional del Ecuador, Ecuador

Autor para correspondencia: diegopqc@ hotmail.com; santiagonajera@gmail.com

Fecha de recepción: 17 de septiembre de 2020 - Fecha de aceptación: 06 de noviembre de 2020

\section{Resumen}

El presente trabajo tiene como principal objetivo desarrollar una herramienta de control y gestión para el inventario de producto terminado para una empresa industrial con la ayuda de herramientas tecnológicas como Excel y el software R Studio. Por tal razón, se analizarán las variables que intervienen o afectan directa e indirectamente al inventario como: datos históricos de venta, datos atípicos de ventas, pronóstico de demanda futura, modelo de proyección, tiempos de reabastecimiento, nivel de servicio, stock de seguridad, inventario promedio e inventario óptimo. Además, el modelo de optimización del inventario está basado en el análisis estadístico de datos como: coeficientes de variación, desviación estándar, valores mínimos y máximos y cuartiles. Debido a la confiabilidad de los datos e información estratégica de la empresa, el presente estudio tiene sus limitaciones y por lo tanto se generan supuestos que serán detallados para que la herramienta a futuro pueda ser utilizada, evaluada y mejorada según amerite.

Palabras claves: optimización; pronóstico; demanda; inventarios; datos atípicos.

\begin{abstract}
The main objective of this work is to develop a control and management tool for the finished product inventory of a company with the help of technological tools such as Excel and R Studio software. For this reason, the variables that intervene in the inventory control will be analyzed, such as: historical sales data, atypical sales data, forecast model, lead time, service level, safety stock, average inventory and optimal inventory. In addition, the inventory optimization model is based on the statistical analysis of data such as: coefficients of variation, standard deviation, minimum and maximum values, and quartiles. Due to the reliability of the data and strategic information of the company, this study has its limitations and therefore assumptions are generated that will be detailed so that the tool in the future can be used, tested and improved as necessary. Keywords: optimization; forecast; demand; inventory; outliers.
\end{abstract}




\section{Introducción}

\section{Antecedentes}

La importancia del inventario en una empresa es debido a que este permite la fluidez de las operaciones ante la incertidumbre que existe entre oferta y demanda (Sharma 2017). Así mismo podemos decir que el inventario es dinero, por lo que es necesario entender el impacto del mismo en la empresa para mejorar la gestión (Muller 2003). En este sentido, una de las herramientas más utilizadas para gestionar el inventario cuando la demanda es predecible, es el modelo de la cantidad económica del pedido EOQ (Silver y Pyke, 2016).

El presente trabajo propone una herramienta de gestión de inventario, mediante el análisis de la variabilidad de las ventas a través del tiempo como indicador de la existencia de la incertidumbre en el proceso de manejo de la cadena de suministro. Incertidumbre que es la base para la correcta decisión de la cantidad de inventario optima que se debe de manejar en la empresa para balancear producción y ventas. Es importante tomar en cuenta, que no existe un modelo perfecto que logre capturar todas las variables del mercado; por esta razón, el modelo debe mantenerse lo más simple, dado que mientras más complejo se vuelve el modelo aumenta el costo de control y ejecución (Silver, 2017).

\section{Definición del problema}

\section{Enunciado del problema}

El presente trabajo asume la existencia de un desbalance entre el costo del inventario en comparación con el nivel de servicio ofertado a los clientes, debido a la falta de procesos y herramientas de gestión y manejo del inventario.

\section{Propósito del estudio}

Proponer una herramienta para la optimización de inventario, mediante un correcto análisis del comportamiento histórico de la demanda, y una depuración de los datos aberrantes dentro de los valores históricos de la misma.

\section{Significancia del problema}

La importancia del presente trabajo radica en que es indispensable para todo tipo de empresas mantener un correcto control de su flujo de caja para asegurar la fluidez de sus negocios (Monteverde 2012). En este sentido, el inventario juega un papel fundamental al ser parte de los activos no líquidos dentro de una empresa (Christopher 2011). Por tal razón, mediante el presente estudio se busca plantear herramientas que ayuden a las organizaciones a un mejor manejo de su flujo de caja.

\section{Naturaleza del estudio}


La naturaleza del estudio tiene como principal propósito ser prescriptiva a través de un proceso cuantitativo como se describe en (Creswell, 2002), mediante análisis estadísticos generar conocimiento y posterior a eso, realizar la interpretación de los datos mediante una lógica deductiva con resultados aplicables.

\section{Limitaciones}

La principal limitación de este estudio es que la demanda y la producción responden a un sinnúmero de variables que impiden ser modeladas al cien por ciento mediante un modelo matemático; razón por la cual, el modelo planteado es una abstracción de la realidad.

\section{Delimitaciones}

En el presente trabajo se asume que el comportamiento de la demanda es un comportamiento normal, y por tanto puede ser modelado mediante el uso de esta distribución. Sin embargo, para aquellos casos en que la demanda no tenga una distribución normal, se eliminará los valores a típico, y se asumirá que la data restante tendera a tener una distribución del tipo normal.

El trabajo de tesis, consiste en realizar un modelo de gestión del inventario mediante un análisis estadístico de sus ventas históricas y en base a teorías existentes desarrolladas por expertos académicos y del área de la administración y gestión de empresas. Este modelo será aplicado a todos los códigos del inventario que forman parte del producto terminado de la empresa de manera generalizada, sin considerar ningún tratamiento o análisis específico para ningún producto.

Además, en el presente estudio, se desarrollarán dos herramientas, una en Microsoft Excel donde se manejará el modelo de gestión y la segunda en Rstudio, la misma que contará con un código de programación para el cálculo del pronóstico de la demanda, donde de la misma manera que la herramienta desarrollada en Excel, el modelo empleado para el pronóstico de la demanda también será una generalización para toda la base de datos.

\section{Objetivos}

\section{Objetivo General}

Diseñar un modelo para gestión y optimización del inventario de producto terminado con el software Microsoft Excel y R Studio.

\section{Objetivos Específicos}

- Determinar las variables clave y como se relacionan las mismas para alcanzar un balance entre los costos del inventario y el nivel de servicio ofertado por la empresa.

- Determinar los aspectos y las variables que la empresa puede mejorar para trabajar en la optimización de su inventario. 
- Determinar si la herramienta de control y gestión del inventario es apta y eficaz para ser usada en la realidad de la operación de la empresa.

\section{Marco teórico}

La incertidumbre de la demanda, implica que las empresas deben asignar recursos para la toma de decisiones del inventario y en su planificación de producción, dado que deben adquirir bienes y materias primas en previsión de las ventas futuras y por otro lado en la planificación de la producción, cierta capacidad debe comprometerse para producir productos y cumplir con la venta futura. Por lo que esta gestión del inventario y de la planificación, requiere un análisis de pronóstico de la demanda y su variabilidad casi siempre será un factor muy significativo para la gestión del inventario (Silver, 2017).

Por otro lado, la mayoría de las empresas no entienden completamente la complejidad de la gestión del inventario y prefieren confiar en lo que se denomina la toma de decisiones de manera heurística o lo que es lo mismo, la toma de decisiones basadas en la experiencia de sus trabajadores o equipo de planificación en lugar de manejar métodos de inventario matemáticamente sólidos. No hay que olvidarse, que el inventario existe para mitigar la incertidumbre en la demanda y los objetivos de la gestión del inventario son: mantener costos bajos y mantener un alto nivel de servicio al cliente; por lo que generalmente las empresas tienen un alto capital involucrado en los inventarios y con mejores herramientas es posible reducir los costos del mismo sin afectar niveles de servicio (Grob, 2019).

La optimización del inventario requiere un análisis con cálculos donde intervienen muchas variables como: demanda, clientes, nivel de servicio, tiempos de reabastecimiento, costos, localidades, regiones, y capacidad de producción. Pero también es crucial el conocimiento y la comunicación a lo largo de toda la cadena de suministro para reducir el efecto látigo, caso contrario la optimización será imposible de mantenerla (Davis, 2016).

\section{Marco conceptual}

\section{Inventario}

Son esas existencias o artículos utilizados para: (a) apoyar la producción (materias primas y artículos en proceso), (b) actividades de apoyo o secundarias (mantenimiento, reparación y suministros operativos) y (c) servicio al cliente (productos terminados y repuestos) (APICS, 2015). El inventario puede ser algo tan simple como una botella de vidrio o un desinfectante como parte del programa de limpieza de un edificio o algo aún más complejo como las materias primas utilizadas en el proceso de fabricación (Muller, 2003).

\section{Demanda}

Es la necesidad de un producto o componente en particular. La demanda podría provenir de cualquier fuente (por ejemplo, un pedido o pronóstico de un cliente, un requisito de trasplante, una solicitud de almacén de sucursal para una parte de servicio o la fabricación de otro producto) 
(APICS, 2015). También la demanda de bienes terminados se la conoce como "independiente", mientras que la demanda de artículos en el mundo manufacturero es "dependiente" y comprender estas distinciones ayudará a pronosticar las necesidades de adquisición (Muller, 2003).

\section{Lead Time}

Es el tiempo de entrega LT que se tarda en cumplir un pedido, es decir, desde el momento que se realiza el pedido hasta que las mercancías estén disponibles para cumplir con la demanda. Por lo tanto, el tiempo de entrega LT, incluye el tiempo de transporte y algunos retrasos que pueden generar actividades intermedias como la inspección y almacenamiento (Grob, 2018). Es conveniente pensar que el tiempo de reabastecimiento está compuesto por cinco componentes distintos: (a) tiempo administrativo, (b) tiempo de tránsito al proveedor, (c) tiempo del proveedor, (d) tiempo de tránsito de regreso al punto de almacenamiento o recepción y (e) tiempo de recepción del pedido (Silver, 2017).

\section{Pronóstico - Forecast}

El pronóstico es una tarea estadística común en los negocios, la misma que ayuda en la toma de decisiones sobre la programación de producción, transporte y personal, y proporciona una guía para la planificación estratégica a corto y largo plazo mediante previsiones del futuro con la mayor precisión posible a través de datos históricos y conocimiento del mercado (Hyndman, 2018).

Los pronósticos pueden basarse en una combinación de una extrapolación de lo que se ha observado en juicios pasados (lo que llamamos pronósticos estadísticos) e informados sobre eventos futuros. La información de eventos futuros puede incluir: (a) pedidos confirmados o acuerdos comerciales confirmados, (b) envíos planificados previamente entre ubicaciones, (c) el uso planificado previamente de piezas de servicio en mantenimiento preventivo y (d) conocimiento general del mercado (Silver, 2017).

\section{Inventario de Seguridad - Stock de seguridad}

El stock de seguridad o inventario de seguridad de los productos dependerá de tres aspectos: tiempo de reabastecimiento o lead time, variabilidad de la demanda, y nivel de servicio requerido. Es posible reducir el nivel de servicio requerido mediante conocimiento del mercado sobre las necesidades y expectativas del cliente (Silver, 2017). El stock de seguridad debe establecerse principalmente en función del cliente y de su inestable demanda, así como la fiabilidad de compra o fabricación y tiempos de entrega. Los factores adicionales que también deben tenerse en cuenta al establecer niveles de stock de seguridad incluyen: (a) la clase o tipo de inventario del artículo principal (A B C), (b) criticidad del uso final, (c) ubicación del cliente, (d) facilidad de fabricación, (e) espacio de almacén, (f) material peligroso, (g) diseño del producto y (h) uso de repuestos (IOMA, 2002).

\section{Nivel de servicio}


Las especificaciones del servicio deben ser el nivel de servicio mínimo que el usuario está dispuesto a aceptar. Se examinarán los requisitos de los usuarios finales para determinar cuáles son los beneficios de niveles de servicio y qué nivel se justifica en función de los costos (Hiles, 2002). También, el nivel de servicio puede ser especificado a priori, bajo políticas empresariales estratégicas para ciertos períodos como una probabilidad de cumplimiento, mediante la elección del factor $\mathrm{Z}$, donde $\mathrm{z}$ es un factor elegido adecuadamente de la tabla normal estándar y aunque la justificación para seleccionar z no es intuitiva, el óptimo puede calcularse utilizando un programa dinámico que requiere muchos cálculos computacionales (Graves, 1993).

\section{Desarrollo}

\section{Análisis preliminar}

Se determinó que los datos recopilados de la empresa deben ser depurados, debido a que varios reportes de ventas corresponden a productos de estrategia denominada bajo pedido o make to order; por ende, estos productos se reportan durante largos períodos de tiempo con una venta en cero, es decir, no han tenido un pedido que active su fabricación y saldrán del estudio. También, se encontraron reportes de venta con datos en negativo que corresponden a devoluciones o notas de crédito y estos serán reemplazados con valores en cero solo para efectos matemáticos dado que no se puede vender en negativo.

\section{Clasificación ABC}

No es recomendable dar el mismo tratamiento a todos los productos del inventario por lo que se sugiere una clasificación $\mathrm{ABC}$ en base a la ley de Pareto. Los SKU son separados en categorías de la siguiente manera: los categorizados con "A" representan los elementos más populares y de mayor movimiento, con letra "B" se representan los elementos "pocos vitales" y finalmente con la letra " $\mathrm{C}$ " los productos que se mueven lentamente. Esta sería una clasificación en base a movimiento de ventas y la Ley de Pareto (IOMA, 2002).

\section{Determinación del nivel de servicio}

Como se mencionó en el marco conceptual del presente trabajo, el nivel de servicio está relacionado con la probabilidad de quedarnos sin inventario ante la variabilidad e incertidumbre de la demanda mediante la curva de Guass y emplearemos este concepto para determinar el nivel de servicio que más se ajuste a la realidad de la operación de la empresa.

Cabe mencionar también, que lo óptimo no es manejar un nivel de servicio del 100\%, sino trabajar sobre el pronóstico de la demanda para conocer aspectos como: estacionalidad (temporadas), ventas atípicas, promociones, tendencia, compromisos comerciales, proyectos e inteligencia del mercado, que ayudan a determinar de una manera más precisa el nivel óptimo de inventario requerido para una fecha futura. 


\section{Figura 1}

Relación del nivel de servicio con la variabilidad de la demanda

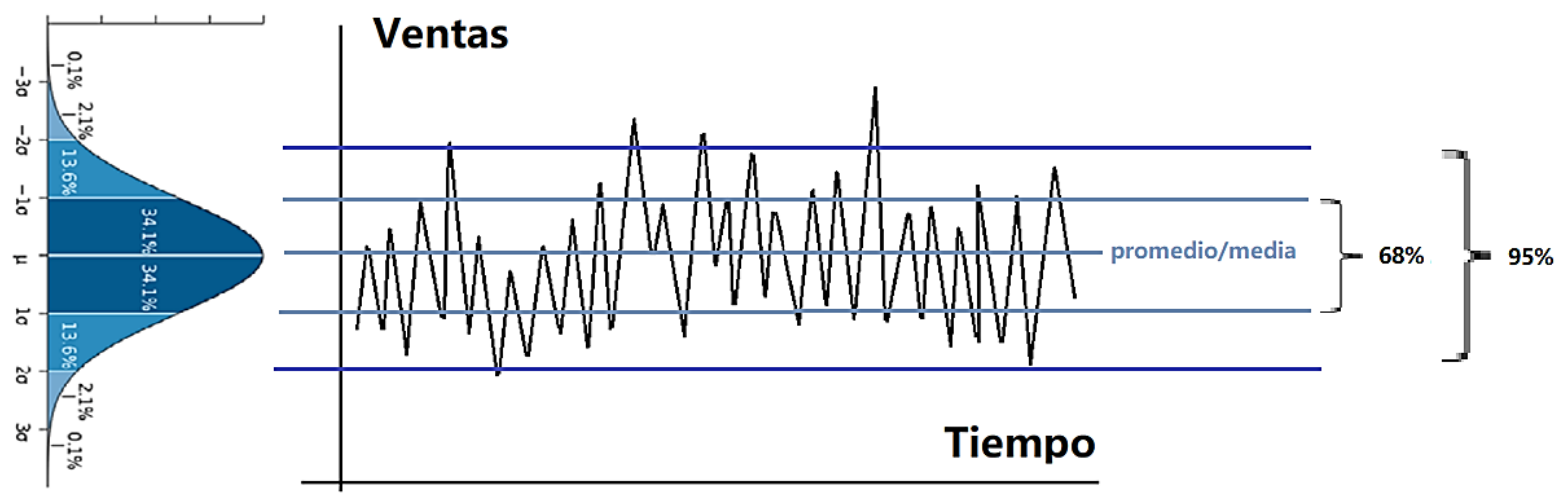

Tampoco debemos olvidar que, a mayor nivel de servicio, mayor nivel de inventario y por ende tendremos un costo del inventario aún mayor. Como punto de partida, y por decisiones empresariales, el modelo manejará $85 \%$ de nivel de servicio.

\section{Elección y ajuste del modelo}

Se han desarrollado varios modelos para el control de inventarios, los mismos que están clasificados por aspectos como: Tiempo de revisión, naturaleza de la demanda, tiempo de reabastecimiento, número de ubicaciones, vida útil de los elementos, etc. (Shenoy, 2018).

Como lo sugirió (Silver, 2017) el modelo de gestión del inventario debe adaptarse a la realidad de la empresa y debe ser consistente con la estrategia y las políticas corporativas de la misma dado que estos aspectos están por encima de cualquier modelo matemático; por lo tanto, el modelo que emplearemos será para productos de la estrategia comercial denominada Make to stock o lo que es conocido con el nombre de sistema push y dado que la organización ha establecido técnicas para pronosticar sus necesidades de nivel de producto basado en la naturaleza de las características de la demanda de esos artículos, nos concentraremos en determinar y elegir el método de pronóstico de la demanda que mejor se ajuste debido a que la optimización del inventario se vuelve una tarea difícil si los modelos de predicción no trabajan conjuntamente con la optimización del inventario y no hay forma que la operación pueda reaccionar si no se conoce la demanda de manera temprana y oportuna.

\section{Análisis de datos históricos}

Es importante analizar los datos históricos, como un análisis estadístico base, para luego poder limpiar y ajustar el modelo para evitar de esta manera posibles distorsiones, y así también poder establecer el modelo de pronóstico de demanda que mejor se ajuste. 


\section{Figura 2}

\section{Comportamiento de Ventas}

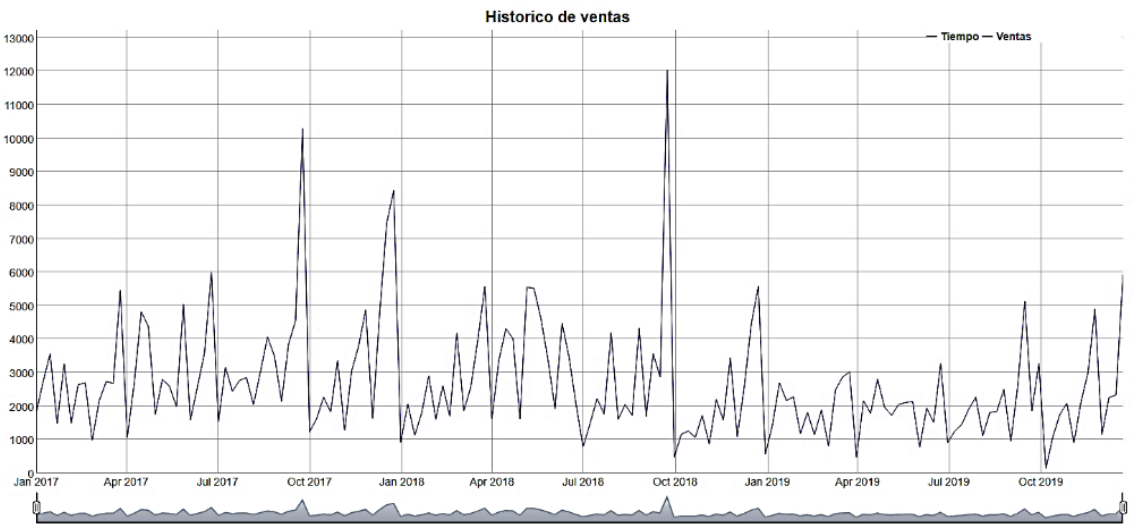

Debido a que se cualquier serie temporal está compuesta de cinco componentes: (a) nivel, (b) tendencia, (c) variaciones estacionales, (d) movimientos cíclicos y (e) fluctuaciones aleatorias irregulares como lo asevera (Silver, 2017); procederemos a analizar estos componentes en la figura 3.

\section{Figura 3}

Descomposición de los datos de una Serie de Tiempo

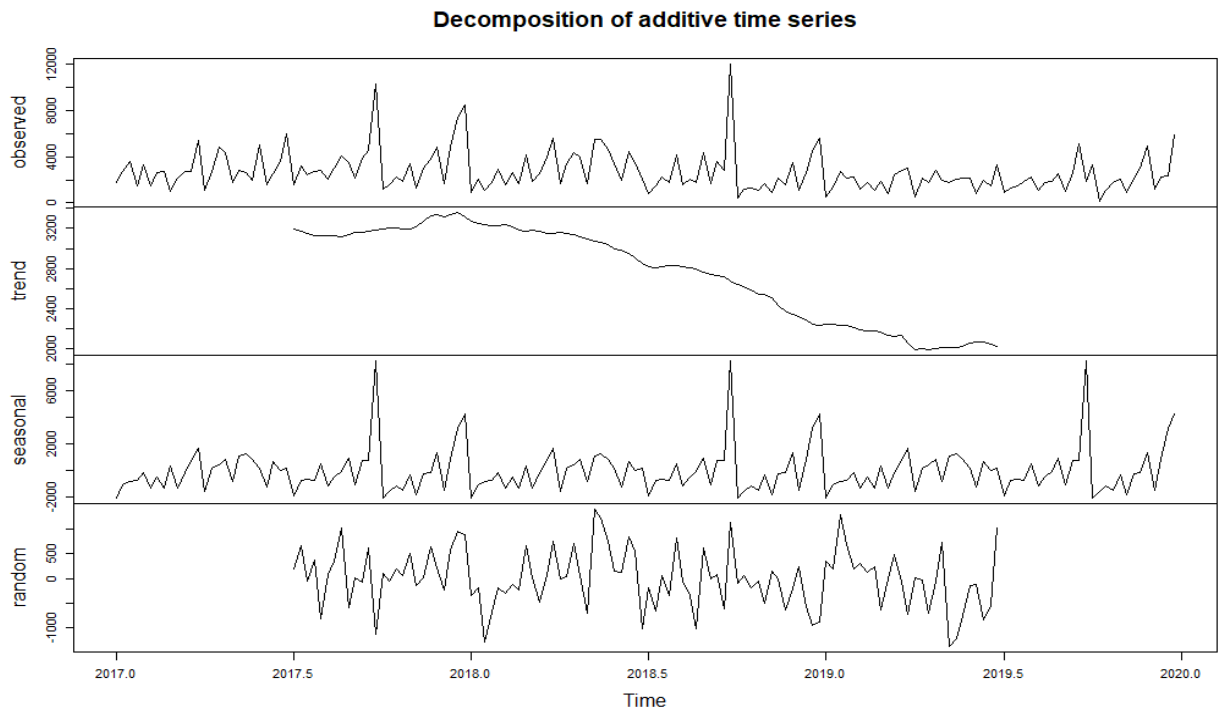

Una vez que hemos analizado gráficamente los datos, podemos incluir algunos parámetros de control estadístico como: la media, el valor mínimo, el valor máximo, el primer y tercer cuartil, lo cual, nos permitirá tener una mejor noción de cómo están nuestros datos históricos. 


\section{Figura 4}

Parámetros de control estadístico

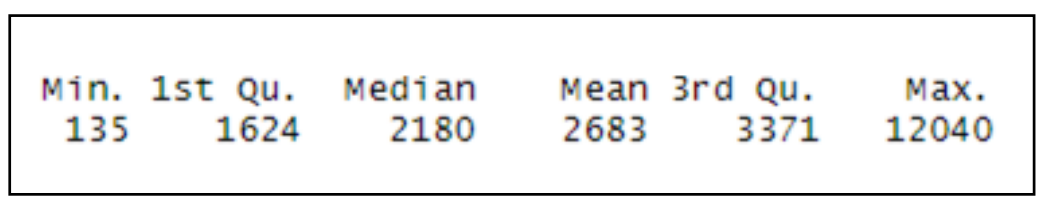

Antes de proceder con los cálculos, para asegurarnos que las fórmulas teóricas del inventario pueden ser aplicadas, realizaremos una comparación gráfica de la serie de datos con la curva de la distribución normal, la misma que nos permitirá conocer si los datos están controlados o no.

\section{Figura 5}

Distribución normal comparada contra gráfico de densidad de los datos

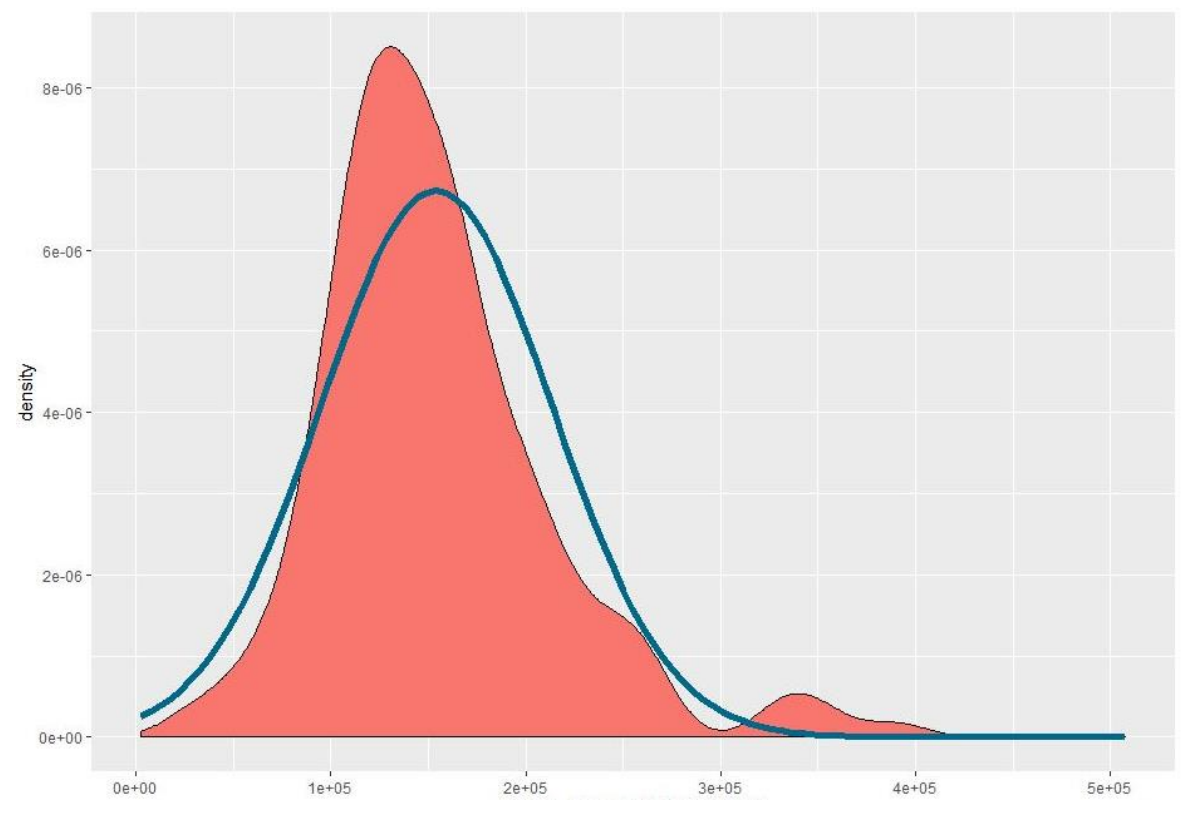

\section{Modelo de pronóstico}

Tenemos algunas formas para pronosticar la demanda como lo son: (a) opinión de expertos, (b) verificación del mercado, (c) análisis cuantitativo y (d) uso de software. Para este estudio nos enfocaremos en el análisis cuantitativo basado en series de tiempo, donde se emplean técnicas para que usan datos de demanda pasada para generar un pronóstico, donde se asume que los patrones de la demanda pasada continuarán en el futuro (ITC, 2006). 
Algunos ejemplos de las técnicas incluyen: (a) línea de tendencia, (b) promedio simple, (c) promedio ponderado, (d) pronósticos ponderados y (e) pronósticos ajustados a la tendencia y estacionalidad.

Gracias al análisis gráfico de la sección anterior, se pudo observar que estos datos podrían ser representados por curvas de complejidad variable con ecuaciones más complicadas como: polinomial, logarítmica, exponencial y de potencia; por lo que, el uso de software se hace imprescindible y emplearemos el modelo de pronóstico Holt-Winters de la librería forecast del software de R Studio, el mismo que pertenece a la familia de suavizado exponencial, con la propiedad que pronostica de manera ponderada las observaciones pasadas, dando relativamente más peso a las observaciones más recientes y a medida que las observaciones envejecen los pesos disminuyen exponencialmente (Hyndman, 2008).

\section{Holt - Winters}

El modelo de pronóstico de la demanda Holt-Winters emplea parámetros como: (a) Beta, para activar el suavizamiento exponencial y (b) el parámetro Gamma que se emplea cuando los datos tienen un componente con estacionalidad. Si al modelo, no se le activa el filtro de suavizamiento exponencial, produce un resultado similar al promedio de los datos como se muestra en la figura 6 .

\section{Figura 6}

\section{Holt-Winter con parámetro Beta en False}

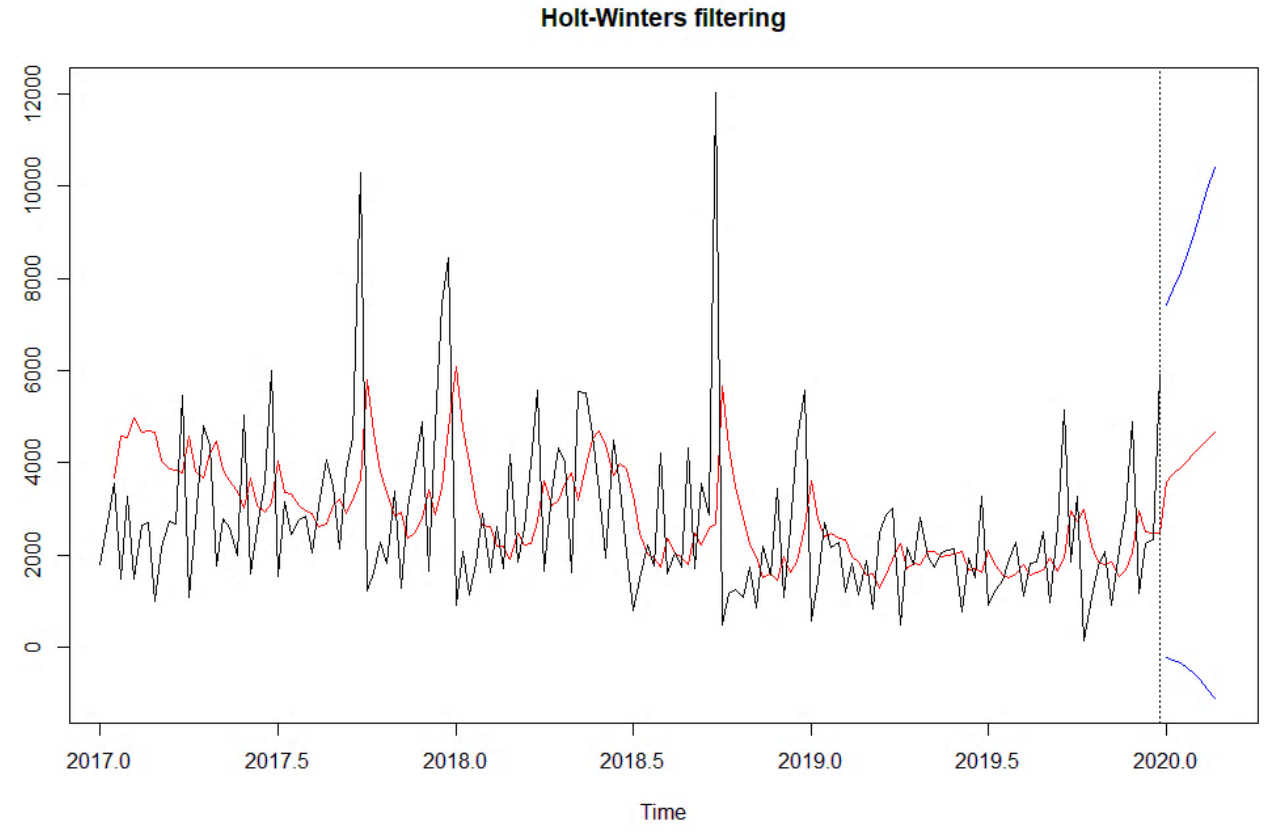


De la misma manera, si desactivamos el parámetro Gamma con false, se produce un resultado similar al mostrado en la figura 6; por lo que hemos podido determinar que el parámetro Gamma debe estar en modo activado o "true" para que el modelo Holt-Winters pueda captar de mejor manera los componentes de estacionalidad como se muestra en la Figura 7 a continuación.

\section{Figura 7}

\section{Holt-Winters con parámetro Gamma en True}

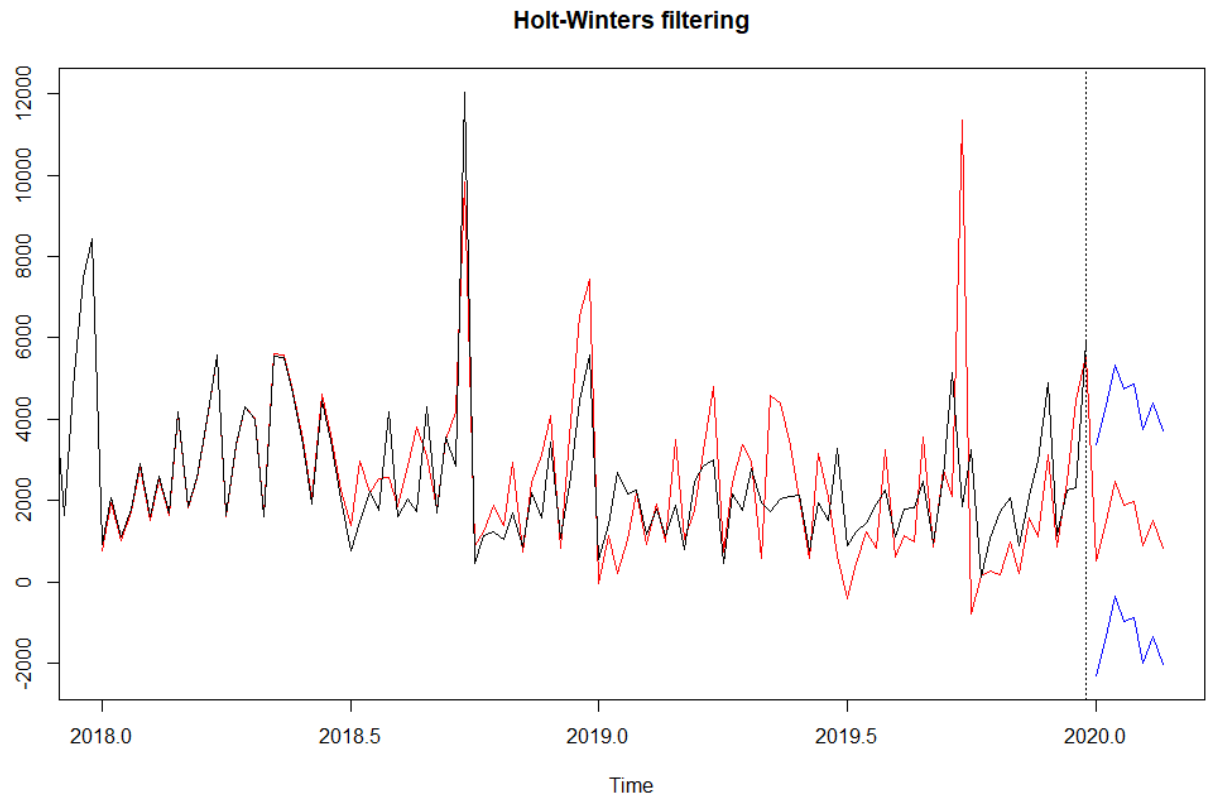

\section{Cálculos}

\section{Factor de lead time}

El factor de lead time lo calculamos a partir de los datos de tiempos de reabastecimiento de la operación de la empresa en estudio, es decir, el factor de lead time se lo calcula a partir del tiempo que la empresa se demora en tener listo un producto para ser entregado al cliente. Dado que los datos del tiempo global de reabastecimiento se encuentran en días, y el inventario se la planifica en semanas; deberemos convertir los días a semanas dividiendo para 5, debido a que la empresa no labora ni sábado ni domingos.

Posteriormente la fórmula para el factor de lead time está dada por la raíz cuadrada del tiempo de reabastecimiento o Lead time.

$$
\text { FactorLeadTime }=\sqrt{(\text { LeadTime })}
$$




\section{Factor de nivel de servicio}

El factor de nivel de servicio nos protege de la variabilidad de la demanda como se analizó preliminarmente, donde el factor de nivel de servicio corresponde al factor $\mathrm{Z}$ de la distribución normal o gráfica de Gauss y su cálculo se lo determina mediante la fórmula de la distribución normal inversa del nivel de servicio. Este valor está relacionado con el número de desviaciones estándar a considerarse.

\section{FactorNiveldeServicio $=$ Distribución normal inversa $($ Nivel de servicio $)$}

Dado que se determinó que el nivel de servicio será del 85\%, si aplicamos la fórmula anterior, obtendremos un valor de factor de Nivel de servicio será igual a 1.

\section{Stock de seguridad}

El stock de seguridad está determinado por tres factores: (a) la desviación estándar de los datos, (b) el factor de lead Time y (c) el factor de nivel de servicio, como se muestra en la siguiente formula:

Stock de seguridad = Desviación Estandar $*$ FactorLeadTime $*$ FactorNiveldeServicio

El factor de Lead Time nos protege contra los posibles imprevistos del reabastecimiento, mientras que el factor del nivel de servicio nos protege de la incertidumbre en la demanda y la variabilidad de la misma. Si los datos fluctúan mucho unos entre otros, mayor será la desviación estándar y por ende mayor cantidad de inventario de seguridad será requerido para cubrir dichas fluctuaciones.

\section{Inventario promedio}

El inventario promedio también conocido como el inventario de ciclo, matemáticamente para este caso estaría dado por la siguiente ecuación:

$$
\text { Inv. Promedio }=\frac{\text { Stock Máx. }- \text { Stock de seguridad }}{2}
$$

Dónde: El stock máximo estaría dado por el dato máximo observado en la serie de tiempo. 


\section{Inventario óptimo}

Una forma válida para calcular el inventario óptimo futuro, está relacionado con las fórmulas del modelo del vendedor de periódicos. El mismo que se establece de la siguiente manera:

\section{Inv. Óptimo $=$ Pronóstico + Desviación estandar $*$ FactorNiveldeServicio}

Relacionando esta ecuación con el modelo del EOQ, se puede deducir, que el inventario óptimo está dado por lo que se pronostica vender a futuro más el stock de seguridad. Si se conocieran las ventas promedio del día, también podríamos aplicar otras fórmulas válidas para el cálculo del inventario óptimo.

\section{Resultados}

\section{Ajuste del modelo}

Los datos arrojados a priori por el modelo, se muestran a continuación en la Tabla 1.

\section{Tabla 1}

Nivel de inventario sin ajuste del modelo (unidades)

\begin{tabular}{ccc}
\hline Familia & Stock seguridad & Inventario promedio \\
\hline Regional uno & & \\
Familia 1 & 71104 & 171540 \\
Familia 2 & 5696 & 13737 \\
Familia 3 & 966 & 2173 \\
Regional dos & & \\
Familia 1 & 33574 & 55612 \\
Familia 2 & 2388 & 4020 \\
Familia 3 & 610 & 963 \\
Total & $\mathbf{1 1 4 3 3 8}$ & $\mathbf{2 4 8 0 4 7}$ \\
\hline
\end{tabular}

Estos son los valores de la suma del inventario promedio y del stock de seguridad para tres líneas de productos (familias) de la empresa. Por lo que, luego de analizar el nivel del 
inventario sugerido en primera instancia por el modelo, se vio necesario ajustarlo quitando datos atípicos o los denominados outliers, debido a que estos valores agregan distorsión a las series de tiempo y por lo tanto el inventario "optimo" sugerido en primera instancia es muy alto.

Si tomamos como ejemplo el producto con mayor movimiento en ventas, tenemos que los datos están en un rango de 0 a 12000 unidades como se muestra en la figura 8. Cuando eliminamos datos atípicos o picos de venta altos, tenemos que el nuevo rango de datos es de 0 a 6000 unidades como se muestra en la figura 9.

\section{Figura 8}

Rango de datos sin depuración de outliers

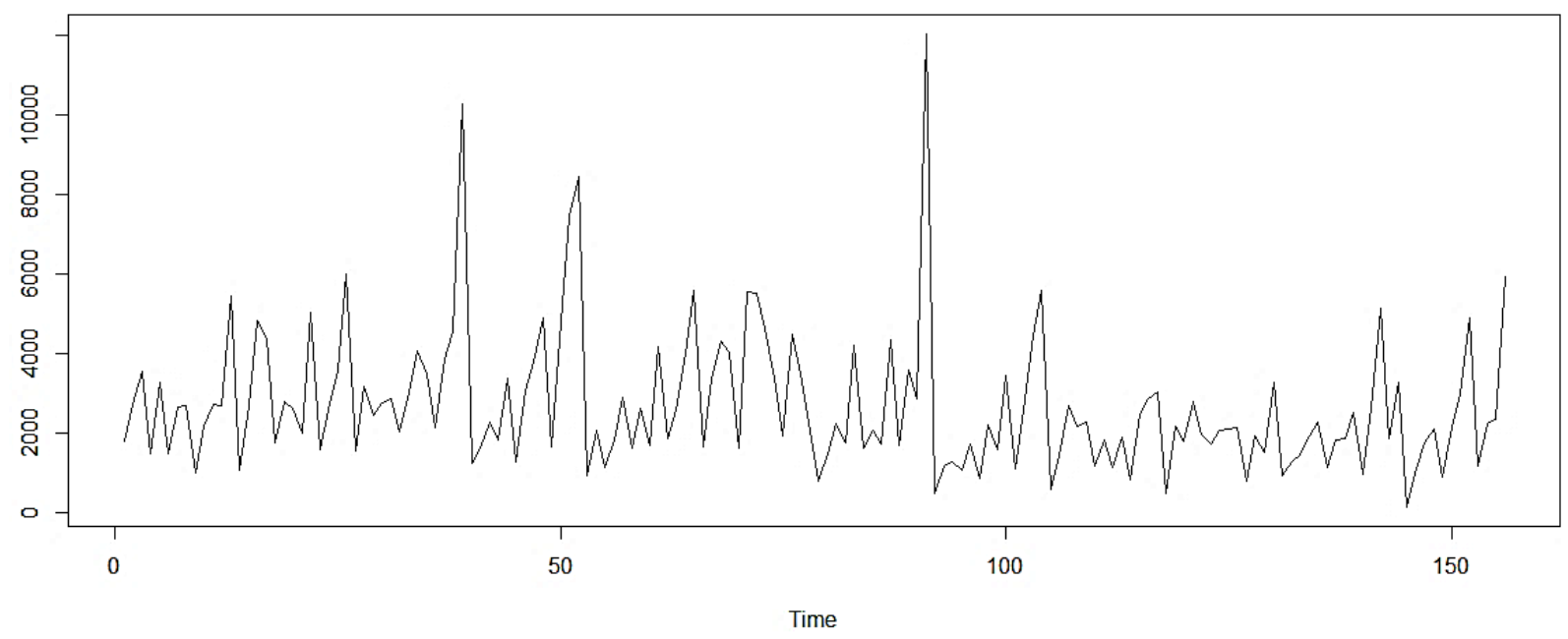

Figura 9

Rango de datos con depuración de outliers

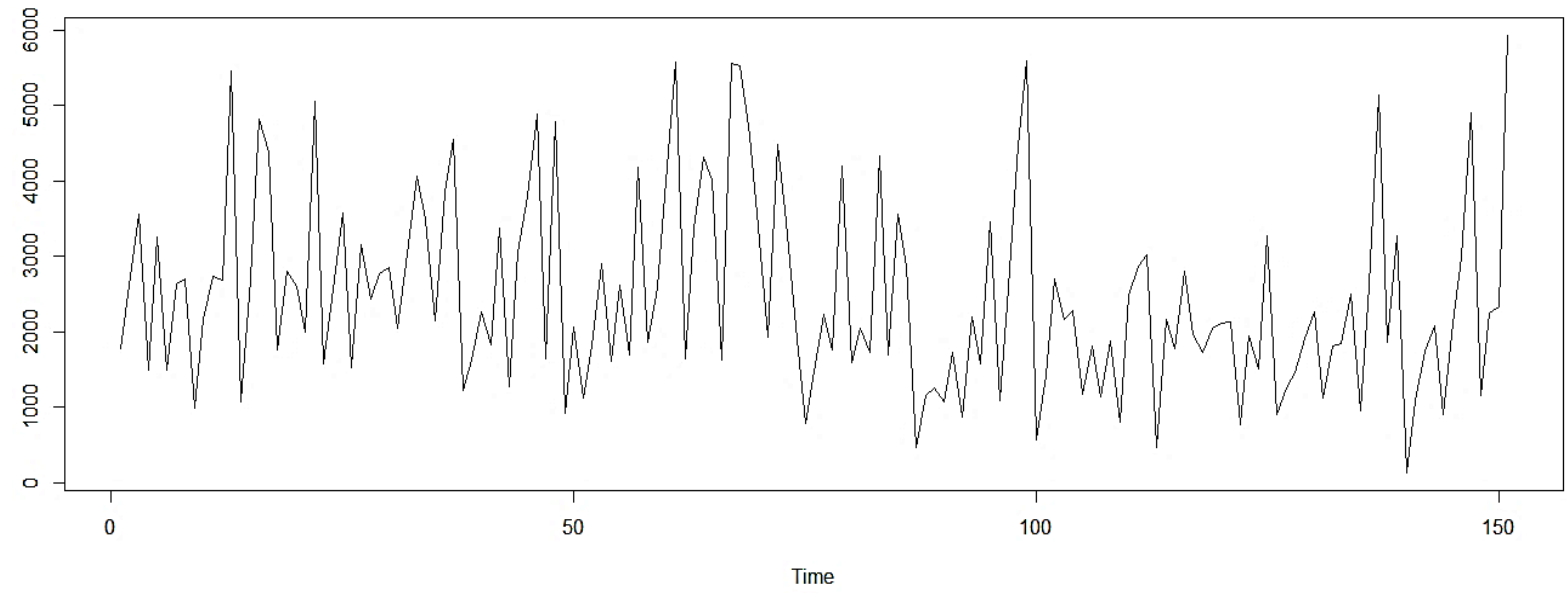


Si calculamos nuevamente los parámetros de control estadístico de: la media, el valor mínimo, el valor máximo, el primer y tercer cuartil, mostrados anteriormente en la figura 10; podremos calcular el porcentaje en el cual han variado estos valores.

\section{Figura 10}

Nuevos parámetros de control estadístico

\begin{tabular}{|rrrrrr|}
\hline \multicolumn{1}{|c}{} & & & & & \\
135 & 1615 & 2160 & 2479 & 3208 & 5925 \\
\hline
\end{tabular}

Al eliminar las ventas atípicas vemos que el valor de la media del nuevo rango de datos ha disminuido en un $7.6 \%$; y el valor máximo de la nueva serie de tiempo se ha visto afectado con una variación del $50.71 \%$. Esto nos muestra lo importante que es depurar y ajustar el modelo, caso contrario podríamos generar un nivel de inventario afectado por las distorsiones.

A continuación, en la tabla 2, se muestran los valores de la suma del inventario promedio y del stock de seguridad para las tres familias de productos una vez ajustado los datos.

\section{Tabla 2}

Nivel de inventario con ajuste del modelo (unidades)

\begin{tabular}{ccc}
\hline Familia & Stock seguridad & Inventario promedio \\
\hline Regional uno & & \\
Familia 1 & 14866 & 34105 \\
Familia 2 & 1745 & 3850 \\
Familia 3 & 347 & 673 \\
Regional dos & & \\
Familia 1 & 13096 & 29338 \\
Familia 2 & 1272 & 2557 \\
Familia 3 & 284 & 571 \\
Total & $\mathbf{3 1 6 0 9}$ & $\mathbf{7 1 0 9 4}$ \\
\hline
\end{tabular}

Podemos calcular que, al quitar datos atípicos de las series temporales, el nivel de inventario promedio bajó en un 71, $3 \%$, logrando de esta manera un inventario optimizado y 
sano para las condiciones actuales de las operaciones de la empresa. Gráficamente, los datos atípicos se muestran en la figura 11 .

\section{Figura 11}

Boxplot de serie de datos con outliers

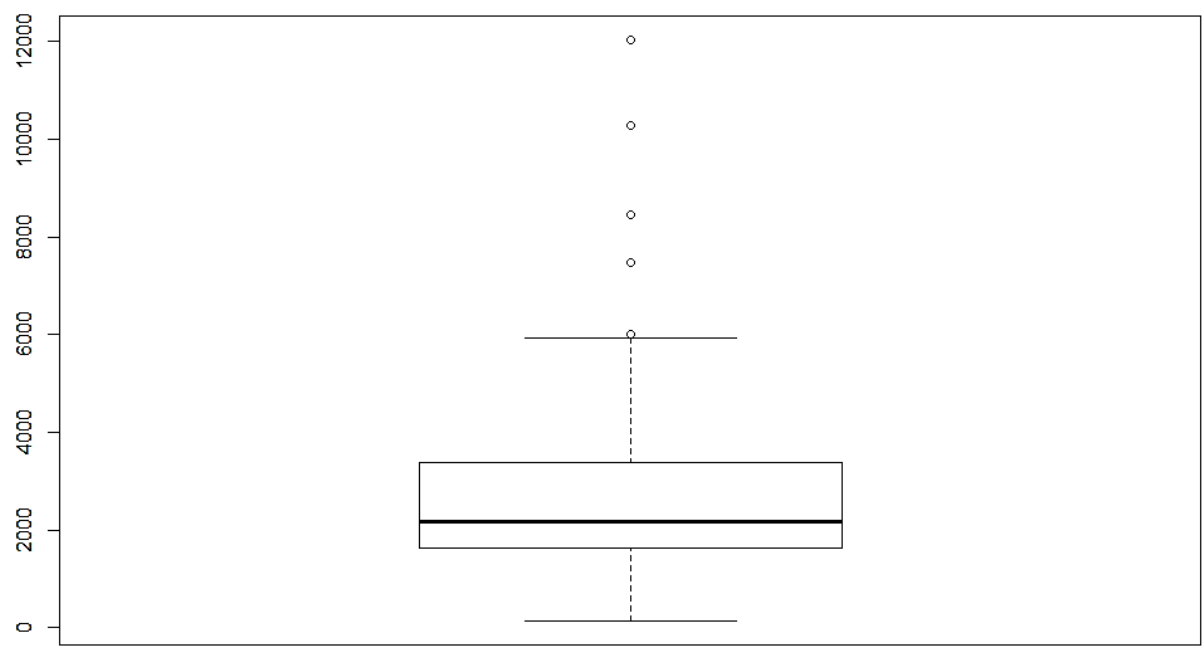

\section{Conclusiones}

Tras haber concluido el trabajo de optimización del inventario de la empresa en estudio, se tienen las siguientes conclusiones:

Se pudo diseñar una herramienta para gestión y optimización del inventario de producto terminado con el software Microsoft Excel y R Studio. La base de datos de los productos con sus respectivos niveles de inventario se lo maneja en una hoja de cálculo, mientras que los valores del pronóstico de la demanda son calculados con el software R Studio y luego son exportados a la hoja de cálculo. Cabe mencionar que el modelo es simple y fácil de usar, ya que esta primera versión será la base de cualquier mejora operativa, donde se le deberá agregar complejidades e información que no fue contemplada por las limitantes de este estudio.

Las variables claves encontradas en el estudio son: (a) nivel de servicio, (b) desviación estándar, (c) tiempos de reabastecimiento, (d) y el pronóstico de ventas. Todas estas se relacionan y sirven para mitigar la incertidumbre de la variabilidad del mercado, basados en una probabilidad de quedarnos sin producto y en tiempos internos y de nuestros proveedores.

Uno de los aspectos para mejorar y que representa una oportunidad para el equipo de trabajo es la depuración de los datos para que ciertas invalidaciones no influencien o afecten el nivel de inventario óptimo del modelo y la principal variable a mejorar es el pronóstico de la demanda, el mismo que con conocimiento del mercado y reglas de decisión heurísticas basadas 
en lógica, producirán una respuesta razonable a situaciones reales más complejas logrando de esta manera que el nivel del inventario sea el más adecuado para la fecha.

Se determinó una forma de medir el grado de efectividad de la herramienta mediante la medición del error, de tres formas distintas que pueden ser empleadas inclusive como indicadores de gestión departamental. Estos indicadores podrían ser los denominados: error cuadrático medio, desviación media absoluta y error porcentual medio absoluto. Cabe mencionar que, por lo general, no se alcanza una optimización absoluta, sino mejoras significativas sobre las operaciones actuales.

\section{Recomendaciones}

Tras haber concluido el trabajo de optimización del inventario de la empresa en estudio, se tienen las siguientes recomendaciones:

Se debe calcular de una manera objetiva el nivel de servicio de los productos, incluyendo datos de costos de excedencias y costos de inexistencias, dado que el inventario es dinero y se lo debe manejar con una perspectiva financiera para entender como este afecta al flujo de efectivo de la empresa y en los balances generales de la misma.

Los datos atípicos encontrados en los históricos de ventas, corresponden principalmente a datos de ventas de temporada alta y cierres trimestrales por que se recomienda siempre limpiar estos datos para no generar alteraciones en el modelo matemático. Así mismo, se debe manejar el pronóstico de la demanda de estas temporadas por separado, dado que el comportamiento es muy alejado de la temporada normal, y se recomienda incluir inteligencia de mercado para el análisis de dichas situaciones.

Se recomienda correr esta herramienta de manera semestral y actualizar los datos de los distintos factores en dicha frecuencia en los productos que no tienen mayor variabilidad en sus reportes de ventas; pero se debe tomar en cuenta que esta no es una herramienta generalizable para todos los productos por lo que, si el giro del negocio o la realidad operativa cambia, se deberá hacer un análisis particular en los productos que ameriten tomando en cuenta el porcentaje de error generado.

Se recomienda a la empresa en estudio, trabajar en la formación de un equipo de trabajo que tenga o desarrolle competencias y conocimientos para la toma de decisiones tanto estratégicas, tácticas y operativas mediante análisis sistemáticos para toda la cadena de suministros y no basar sus decisiones en aspectos subjetivos; por lo que la construcción de herramientas con indicadores de gestión permitirá también al grupo enfocarse en objetivos.

Se podría trabajar en la automatización de las herramientas mediante el uso de software y herramientas de licencia gratuita como el caso del software R Studio, dado que en esta era de la transformación digital, ya no es eficiente que el equipo de trabajo invierta tiempo en generar y recopilar información, sino el tiempo debe invertirse en la toma de decisiones y la generación de 
análisis y conocimiento apoyándose de la reportería de herramientas de inteligencia de negocios o business intelligence.

\section{Referencias bibliográficas}

APICS. (2015). Learning System (Vol. 4.2).

Christopher, M. (2011). Logistics \& supply chain management, Pearson education limited.

Creswell, J. (2002). Research Desing Qualitative, Quantitative, and mixed methods approaches (Vol. 2 ed).

Davis, R. (2016). Demand-Driven Inventory Optimization and Replenishment (Vol. 2nd Edition). New Jersey, USA: John Wiley \& Sons, Inc.

Graves, S. (1993). Logistics of Production and Inventory (Vol. vol 4). Elsevier Science Publishers.

Grob, C. (2018). Inventory Mangement in Multi-Echelon Networks. Wolfsburg, Germany: Springer.

Hiles, A. (2002). The Complete Guide to IT Service Level Agreements. Connecticut, USA: KingWell.

Hyndman, R. (2008). Forecasting with Exponential Smoothing. Clayton, Australia: Springer.

IOMA. (2002). Logistics and Inventory Management. New York, USA: John Wiley \& Sons, Inc.

ITC. (2006). International, Trade Centre - Especificación de Requerimientos y Plan del Suministro. Ginebra, Suiza.

Monteverde, E. C. (2012). Finanzas Corporativas. Con nuevos temas y ejercicios, Cengage Learning Editores S.A. de C.V.

Muller, M. (2003). Essentials of Inventory Management. New York, USA, AMACOM.

Sharma, S. (2017). Inventory parameters, Springer.

Shenoy, D. (2018). Problems \& Solutions in Inventory Management. Springer.

Silver, E. (2017). Inventory and Production Management in Supply Chains. Boca Raton, Florida, USA, CRC Press. 